\title{
INFLUENCE DE LA TAILLE DES GRAINS SUR LE COMPOR'TEMENT DYNAMIQUE DU CUIVRE
}

\author{
G. GAZEAUD et A. LICHTENBERGER \\ Institut Franco-Allemand de Recherches de Saint-Louis, BP. 34, \\ F-68301 Saint-Louis cedex, France
}

\begin{abstract}
Résumé: On a étudié le comportement du cuivre par des essais statiques et dynamiques d'échantillons de différentes granulométries: 20 et $110 \mu \mathrm{m}$. Les essais dynamiques montrent que l'influence de la granulométrie sur la consolidation est moins marquée que dans les essais statiques où la différence apparaît déjà à faible déformation. Avec le même matériau on a recherché l'influence de la granulométrie sur la forme finale d'un projectile formé par explosif (type CGN). Dans un tel essai le matériau est soumis d'une part à la pression de choc élevée transmise par l'explosif (30 Gpa), d'autre part à une déformation plastique importante (plus de $100 \%$ ), et à grande vitesse de déformation $\left(10^{3}\right.$ à $\left.10^{4} \mathrm{~s}^{-1}\right)$.
\end{abstract}

\begin{abstract}
The behavior of copper of fine and coarse grain size (20 and $120 \mu \mathrm{m})$ has been studied by the mean of static and dynamic tests. Dynamic experiments show that grain size influence is less important than in static for which an effect is effective at low strains. With this material, the role of grain size has been tested on the final shape of Explosively Formed Projectiles (EFP). Material is first submit to a high pressure (30 GPa) given by explosive shock wave, large distortions (over $100 \%$ ) and strain rates of $10^{3}$ to $10^{4} \mathrm{~s}^{-1}$.
\end{abstract}

\section{1) Introduction}

La modélisation du comportement dynamique des matériaux nécessite une bonne connaissance dans le domaine des grandes vitesses de déformation et des hautes pressions. L'influence d'une onde de choc a déjà été étudiée, mettant en évidence une augmentation de la contrainte d'écoulement plastique avec la pression de choc. On propose d'étudier la différence de comportement en fonction de la granulométrie du cuivre. On utilise le test d'expansion d'anneau par explosif et on présente une application aux charges génératrices de noyaux avec étude micrographique des projectiles récupérés.

\section{2) Matériau étudié}

\subsection{Caractérisciques physiques}

Le matériau étudié est un cuivre pur du type Cuc2 (OFHC), mis en forme par forgeage à chaud en plaques d'épaisseur $40 \mathrm{~mm}$, par laminage croisé et recuit intermédiaire à 490 degrés. 
$L^{\prime e ́ t a t ~ f i n a l ~ e s t ~ o b t e n u ~ p a r ~ u n ~ r e c u i t ~ a ̀ ~} 490$ degrés $\left(670^{\circ}\right)$ en vue d'obtenir une taille de grains de 30-40 microns (110-120 microns).

La composition chimique, en ppm, est donnée ci-dessous:

\begin{tabular}{|c|c|c|c|c|c|c|c|c|c|}
\hline $\mathrm{O} 2$ & $\mathrm{C}$ & $\mathrm{Ag}$ & $\mathrm{Fe}$ & $\mathrm{S}$ & $\mathrm{As}$ & $\mathrm{Sn}$ & $\mathrm{Pb}$ & $\mathrm{Al}$ & $\mathrm{P}$ \\
\hline 15 & 18 & 13 & 5 & 3 & 1.6 & $<1$ & 0.1 & $<1$ & 1.5 \\
\hline
\end{tabular}

La température de recristallisation, mesurée selon la méthode I.S.L (1), est de $285^{\circ} \mathrm{C}$. La dureté Vickers varie de 49 à 55 pour le cuivre à grains fins et de 37 à 40 pour les gros grains. Les coupes granulométriques sont présentées sur la figure 1. Il faut noter que la coupe 110-120 $\mu \mathrm{m}$ n'est pas très homogène, la taille des grains varie de 80 à $400 \mu \mathrm{m}$.

\subsection{Mesures statiques}

On a effectué des compressions et tractions statiques pour les deux qualités de cuivre. Les échantillons ont été prélevés dans les trois directions afin de vérifier l'anisotropie due aux divers traitements thermo-mécaniques. Les courbes de la figure 2 correspondent à des compressions statiques réalisées à deux vitesses de déformation: $10^{-4}$ et $10^{-2} \mathrm{~s}^{-1}$. Les courbes statiques ont été ajustées par la relation asymptotique:

$$
\sigma_{\text {stat }}=\mathrm{A}+\frac{\left(\mathrm{B} \times \bar{\varepsilon}^{\mathrm{p}}\right)}{\left(\mathrm{C}+\vec{\varepsilon}^{\mathrm{p}}\right)}
$$

Cuivre à grains fins: $\quad \mathrm{A}=70 \mathrm{MPa}, \mathrm{B}=345, \mathrm{C}=0.13$

Cuivre à gros grains: $\quad \mathrm{A}=50 \mathrm{MPa}, \mathrm{B}=430, \mathrm{C}=0.27$

Les courbes de traction statique et compression dynamique de la figure 3 montrent une bonne concordance. Le sens A correspond à des échantillons pris perpendiculairement au laminage et parallèlement pour le sens $B$.

La contrainte à la rupture de $327 \mathrm{MPa}$ est en accord avec d'autres résultats (2). Mais elle est d'environ $50 \mathrm{MPa}$ inférieure pour le cuivre à grosse granulométrie. Les déformations plastiques équivalentes à la rupture sont de $29 \%$ pour le cuivre à gros grains et $37 \%$ pour les fins, correspondant respectivement à des allongements à la rupture de $48 \%$ et $39 \%$. Cette anomalie peut s'expliquer par le rapport entre la taille des grains et celle des échantillons (3).

La loi de Petch s'applique très bien pour le cuivre (fig.4), avec les coefficients suivants:

$$
\sigma_{\mathrm{y}}=\sigma_{0}(\varepsilon)+\frac{\mathrm{K}}{\mathrm{d}^{(-1 / 2)}} \quad \mathrm{K}=11.8
$$

\section{3) Essais dynamiques}

\subsection{Barres d'Hopkinson}

Les essais de compression dynamiques à l'aide d'un système de barres d'Hopkinson conduisent à deux remarques. Le comportement dynamique conserve la hiérarchie entre les tailles de grains pour la valeur de la contrainte d'écoulement plastique, à savoir que pour la granulométrie fine, la contrainte est plus élcvée pour une déformation donnée. D'autre part, 
cet effet tend à diminuer en régime dynamique lorsque la déformation augmente, contrairement aux essais statiques (fig 5).

Lors de la modélisation de charges du type E.F.P, le matériau, avant de se déformer plastiquement, subi un choc d'environ $30 \mathrm{GPa}$, comme l'indiquent les simulations numériques.

On utilise alors un test qui simule ce choc initial (4).

\subsection{Test d'expansion d'anneau}

L'anneau de cuivre entourant le cylindre d'acier est mis en vitesse par l'onde de choc qui traverse ce cylindre. L'anneau se déforme ensuite plastiquement sous l'action d'aucune force extérieure. On rappelle les relations qui permettent de relier la contrainte $(\sigma)$, la déformation $(\varepsilon)$, et la vitesse de déformation $(\dot{\varepsilon})$ :

$$
\sigma=-\rho \mathrm{r} \ddot{\mathrm{r}} \quad \varepsilon=\ln \frac{\mathrm{r}}{\mathrm{r}_{0}} \quad \dot{\varepsilon}=\frac{\dot{\mathrm{r}}}{\mathrm{r}_{0}}
$$

Ce test offre de plus l'avantage de pouvoir étudier quantitativement la rupture du matériau testé. Les deux nuances de cuivre ont été testées avec des pré-chocs de 3 à $6 \mathrm{GPa}$, correspondant à des diamètres d'explosif respectifs de 5 à $10 \mathrm{~mm}$. Les enregistrements des vitesses au cours du temps (fig.6) montrent un léger décalage, montrant la sensibilité de la méthode. La remarque faite pour les compressions dynamiques restent valables en traction dynamique, à savoir que la différence entre les contraintes diminuent lorsque la déformation augmente (fig.7).

On a montré (5). que pour le cuivre, le choc initial est important pour les faibles déformations. Dans la présente étude, aucune influence de ce front de pression en fonction de la taille de grain n'a pu être mis en évidence. Par contre, la déformation à la rupture (voir le tableau cidessous) montre que lorsque le niveau de pression augmente, une limite est rapidement atteinte aux alentours de $45 \%$, valeur proche à celles mesurées (43\% sans pré-choc), dans d'autres laboratoires (6). Cette valeur de la déformation à la rupture, indépendante de la taille de grains, est le résultat de deux effets qui se compensent. La limite d'écoulement plastique du cuivre à gros grains étant plus faible, ce dernier tend à se déformer d'avantage. Cet effet est compensé par une rupture précoce due à une plus grande hétérogénéité du matériau.

\begin{tabular}{|c|c|c|c|c|c|}
\hline $\mathrm{P}(\mathrm{GPa})$ & 3 & 4 & 5.5 & 6 & \\
\hline$\varepsilon(\%)$ & $22\left(^{*}\right)$ & 35 & 45 & 45 & Fin \\
& $25\left(^{*}\right)$ & 30 & 45 & 45 & Gros \\
\hline
\end{tabular}

(*): non rupture

Lorsqu'il n'y a pas rupture, les anneaux à grains fins restent très homogènes, tandis qu'ils se forme de nombreuses strictions sur les anneaux à gros grains. La déformation plastique maximale à la rupture, calculée à partir des sections homogènes des fragments et mesurée expérimentalement, est une valeur moyenne.

L'influence de la vitesse de déformation a été menée à partir d'expériences ou la pression d'entrée est constante (4 GPa), et en changeant la section de l'anneau, ce qui a pour effet de faire varier la vitesse de déformation maximale. Les sections sont passées de $1.5 * 1.5$ à $3 * 3$ $\mathrm{mm}^{2}$. La variation de la contrainte d'écoulement plastique est indépendante de la vitesse de déformation, l'écart entre les deux courbes (gros et fins grains) étant constant quelque soit la vitesse (figure 8). 


\section{4) Application aux C.G.N}

Un des objectifs de l'étude du comportement du cuivre est son emploi dans la formation de projectile par explosif. On a donc utilisé le même cuivre que précédemment, en affinant les coupes granulométriques. Les micrographies correspondantes (fig.9) montrent une plus grande homogénéité pour les grains fins $(20 \mu \mathrm{m})$ et une coupe 100-400 $\mu \mathrm{m}$.

Les projectiles formés par explosif sont récupérés à l'aide d'un tube rempli de mousse de densités croissantes. Il est alors possible de les couper pour effectuer des mesures de dureté et des observations micrographiques.

A partir des radiographies des projectiles en vol et après confirmation sur ceux qui ont été récupérés, on constate que les revêtements à grains gros engendrent des projectiles plus allongés. Le rapport longueur sur diamètre est respectivement de 2.8 et 2.2. La partie pleine est plus importante comme l'ont montrées les simulations numériques (7) lorsque la contrainte d'écoulement plastique est plus faible. Ceci confirme que la pression de choc et la vitesse de déformation ont le même effet quelque soit la granulométrie (fig.10)

L'étude micrographique indique une recristallisation complète des échantillons récupérés. En ce qui concerne la structure du matériau, les traces de son histoire sont visibles principalement sur les projectiles à gros grains. Les lignes de déformations (fig.11) sont dues à une différence de recristallisation qui résulte de l'inhomogénéité initiale. En effet les déformations au niveau des grains sont plus importantes qu'avec les grains fins ou ce phénomène est moins visible. $\mathrm{La}$ figure 14 montre que ces lignes résultent d'une recristallisation différente du reste de l'échantillon avec un indice de taille de grains de 11 au lieu de 7 (resp. $6 \mu \mathrm{m}$ et $30 \mu \mathrm{m}$ ). Celle qui est parallèle à la surface du revêtement est probablement due à un début d'écaillage lors du passage du choc initial. Cette ligne n'est visible qu'avec les revêtements à grosse granulométrie.

On peut voir sur la figure 12 une vue détaillée des lignes de déformation induites lors de la formation du projectile. Ces lignes ressemblent à des lignes de cisaillement adiabatiques (8). Des mesures de micro-dureté montrent que ces bandes de cisaillement ne sont pas recristallisées.

Les observations faites à la base de la partie pleine des projectiles apportent un élément de réponse sur l'instant de recristallisation. Les micro-fissures visibles sur la figure 13 constituent le joint des grains recristallisés, indiquant que la recristallisation pourrait avoir eu lieu après la déformation et la fissuration, donc tardive et probablement lors de la récupération dans la mousse.

Des mesures de micro-dureté font ressortir une plus grande homogénéité avec les grains fins, 68 à $70 \mathrm{Hv10}$, contre 53 à $60 \mathrm{Hv} 10$ pour les gros. Dans ce dernier cas, la dureté la plus élevee se situe à la base de la partie pleine du noyau, ce qui explique une ductilité plus faible pour ces revêtements à grosse granulométrie. En effet, ces derniers se cassent alors que ceux à grains fins sont à la limite de rupture.

\section{5) Conclusion}

Le comportement statique et dynamique du cuivre est fortement influencé par sa coupe granulométrique. Les essais statiques font ressortir une augmentation de la différence de contrainte plastique en fonction de la déformation. Cette effet est moins sensibles pour les essais dynamiques, essentiellement lorsque la déformation est précédée d'un choc. On ne constate pas de différences significatives de comportement au choc et à la vitesse de déformation entre les deux coupes granulométriques. 
Les examens micrographiques de noyaux formés par explosif fournissent des renseignements importants sur la nature et la forme des lignes de déformations intenses ainsi que sur l'instant de recristallisation.

Références

11/ LiChTENBERGER,A. 11th Internal Symposium on Ballistics, 9-11 May 89 Bruxelles-Belgium. Vol.2 p103.

12/ FOLLANSBEE,P.S. Metallurgical Applications of Shock wave and High-Strain-rate Phenomena, ed. L.E. Murr,K.P Staudhammer and M.A Meyers (Marcel (Dekker, New-York, 1986) p451-479.

13/ GOURDIN,W.H Shock waves in condensed Matter, Monterey, California, 20-23 July, 1987.

14/ DUJARDin,S. GAZEAUd,G. LiCHTENBERGER,A. Dymat 88, 19-23 Sept. 1988, Ajàccio.

15/ GAZEAUD,G. 3e Symposium International Hautes Pressions Dynamiques, 5-9 juin 1989, La Grande Motte, France.

16) GOURDIN,W.H 4th Mechanical Properties of Materials at High Rates of Strain, Oxford, 19-22 March 1989.

17/ GAZEAUD,G. I.S.L RT 515/84, 1984.

18/ NAI-YONG TANG NIESSEN,P. PICK,R.J WORSWICK,M.J. Material Science and Engineering,A131(1991) 153-160. 

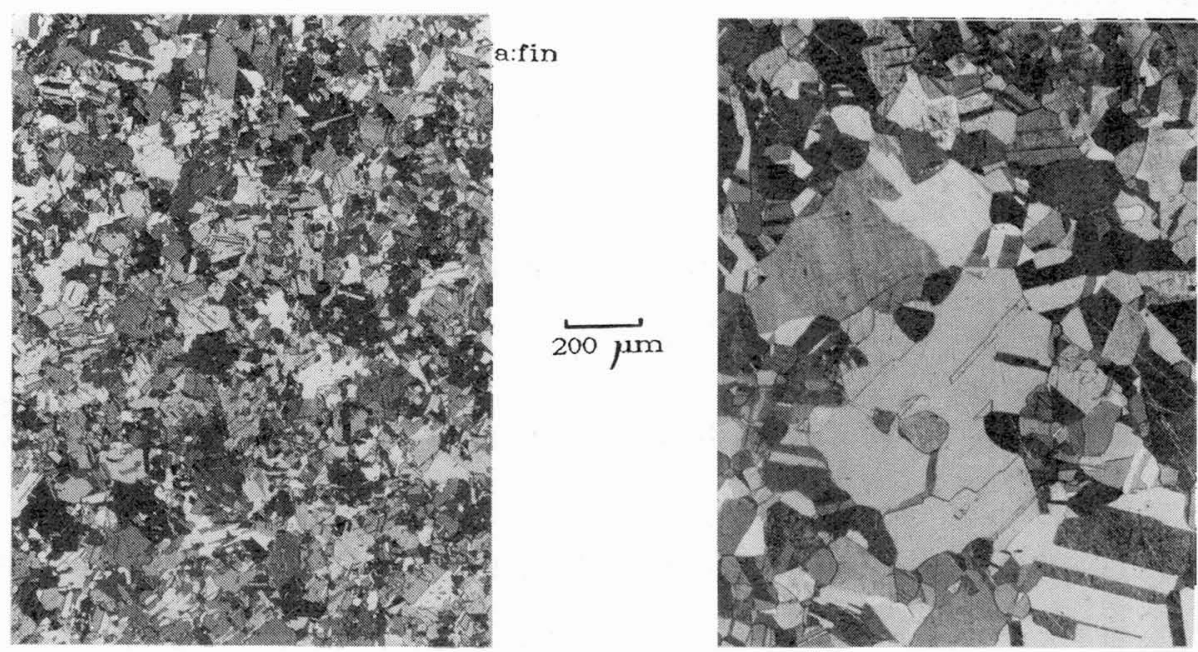

Fig.1 Etat initial

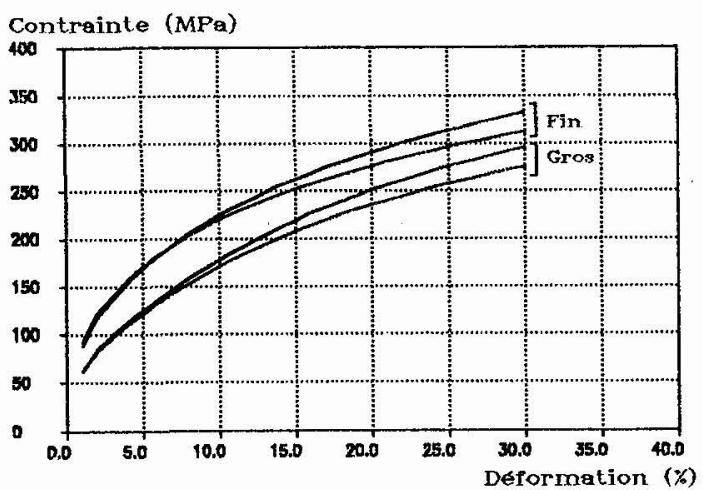

Fig.2 Compression statique

Contrainte (MPg)

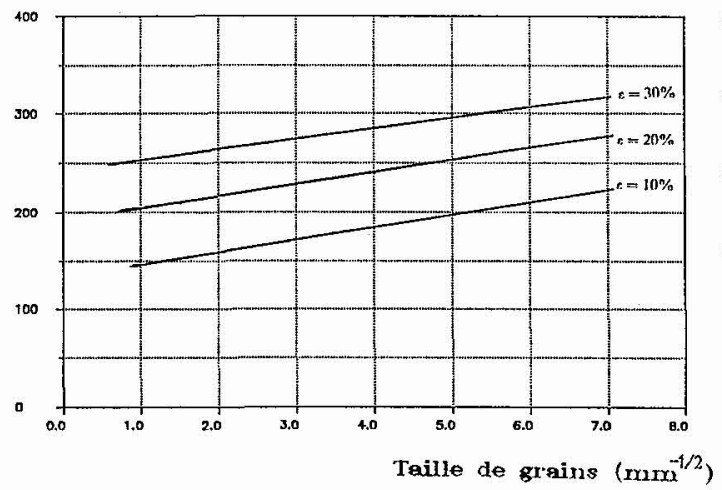

Fig.4 loi de Petelh

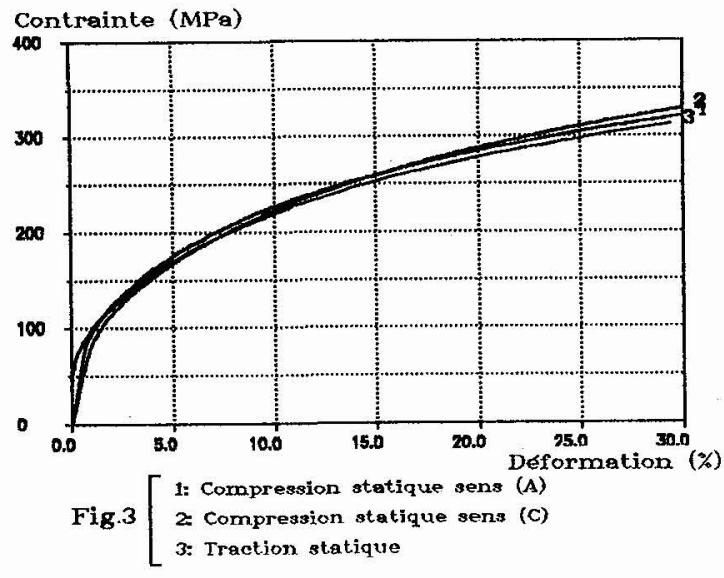

Contrainte (MPa)

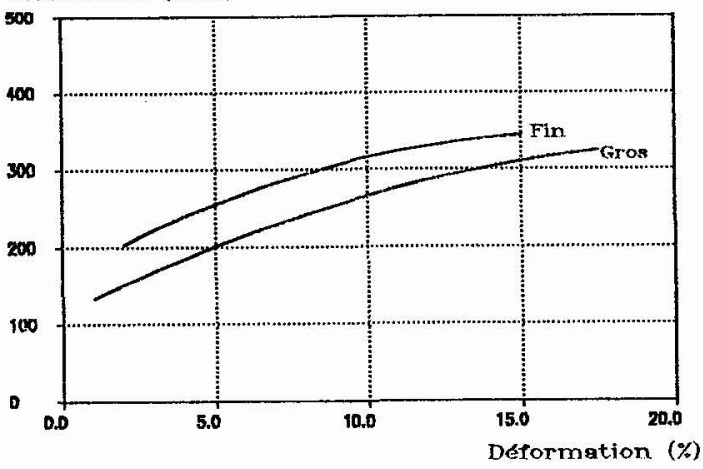

Fig.5 Compression dynamique 


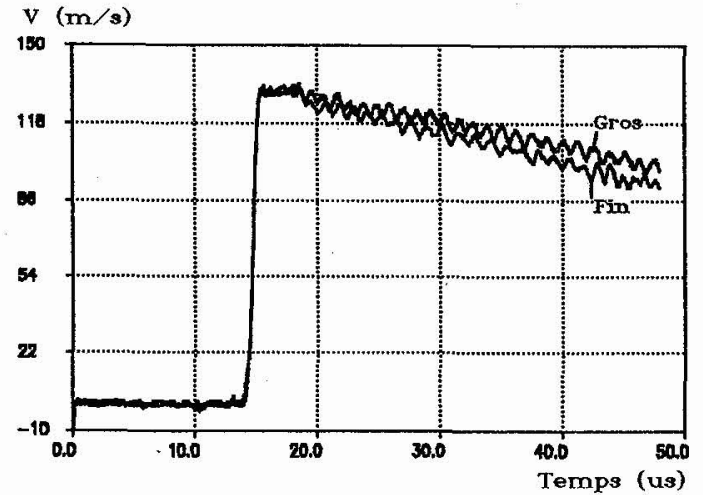

Fig. 6 Vitesse de l'anneau

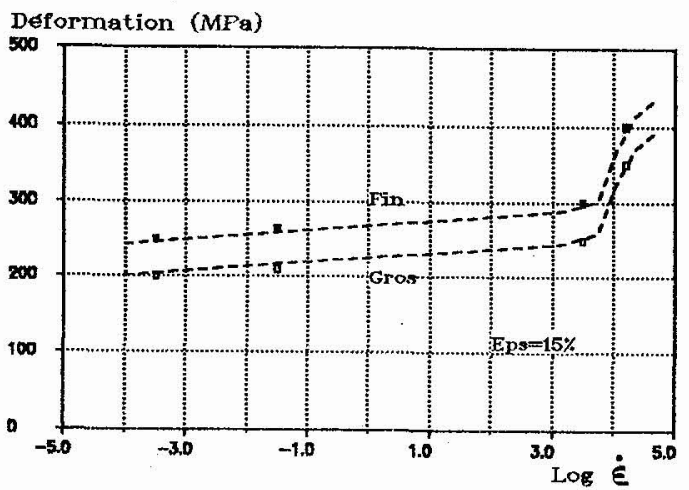

Fig.8 Influence de la vitesse de deformation

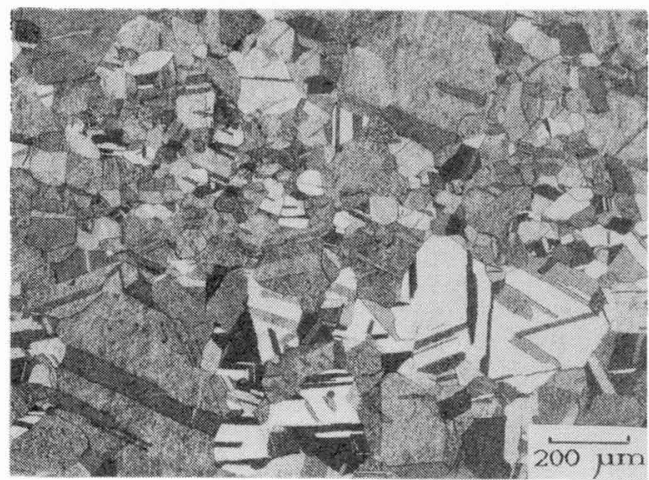

Fig.9b Etat initial: Gros grains

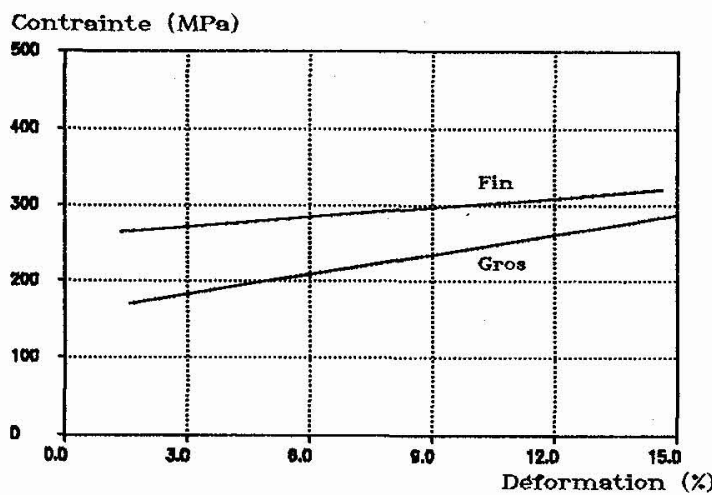

Fig.7 Test d'expansion d'anneau

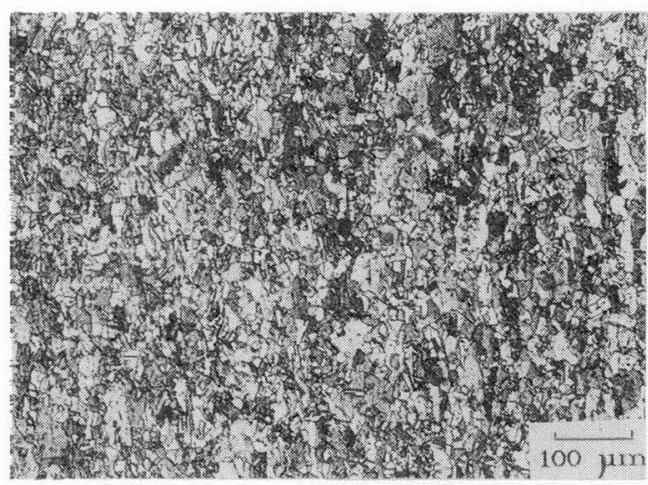

Fig.9a Etat initial: Grains fins
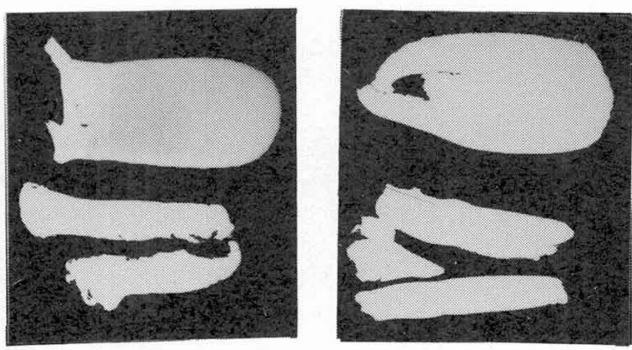

Gros

Fin

Fig.10 Projectiles recuperes 


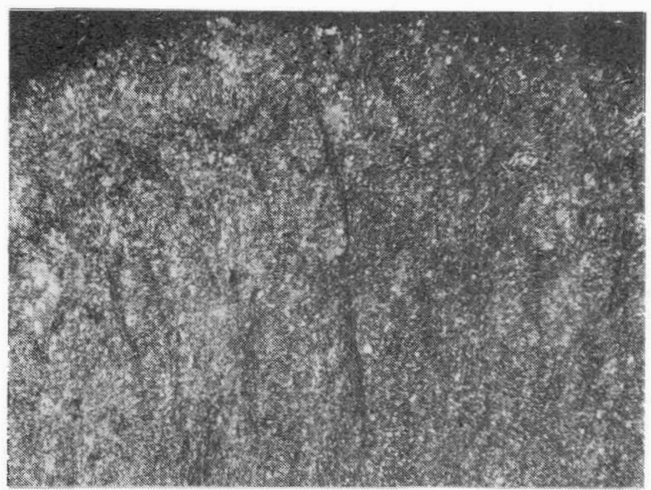

Gros $\stackrel{\mathrm{mm}}{\longrightarrow}$

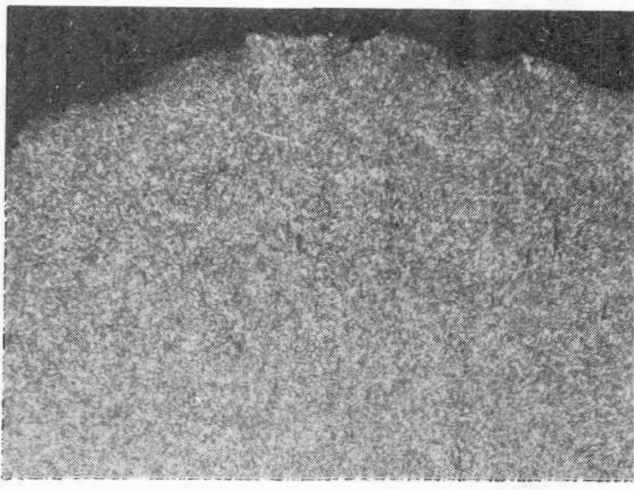

Fin

$1 \min$

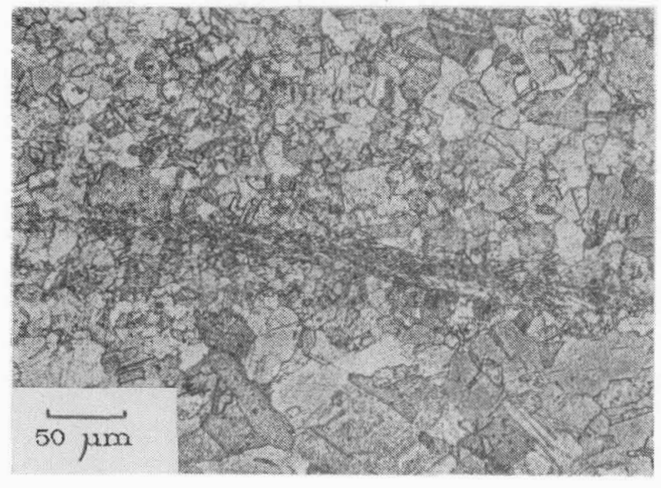

Fig.12 Détail d'une ligıze de deformation

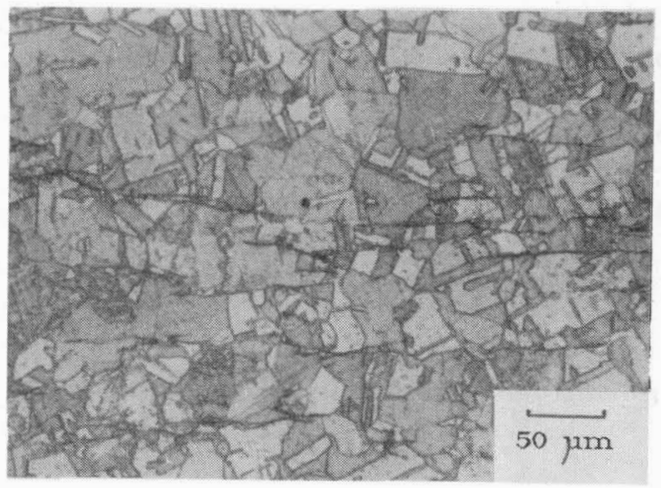

Fig.13 Micro-fissures intergranulaires

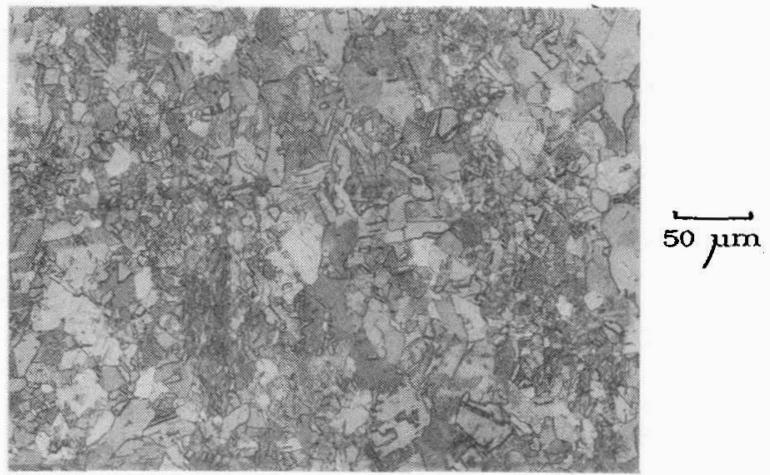

Fig-14 Zones de recristallisations differentes 